\title{
CD38: From Positive to Negative Expression after Daratumumab Treatment
}

\author{
Bonell Patiño-Escobar ${ }^{1}$, Roberto Ramos $^{1}$, Maximo Linares ${ }^{1}$, Angie Mejía ${ }^{1}$, Sebastián Alcalá ${ }^{1}$ \\ 1. Hematology, Instituto Nacional de Cancerología, Bogota, COL
}

Corresponding author: Bonell Patiño-Escobar, bonell32@hotmail.com

\begin{abstract}
CD38 is a glycoprotein expressed at a low level in myeloid and lymphoid tissues. However, it is highly and homogeneously expressed in plasma cells (PC) in multiple myeloma. Daratumumab is a human CD38specific IgG1 antibody available for the treatment of multiple myeloma in Colombia. It has been authorized in relapsed/refractory disease as front-line treatment for non-eligible stem cell transplantation patients by INVIMA (Instituto Nacional de Vigilancia de Medicamentos y Alimentos) that is the regulatory agency. Daratumumab treatment has been associated with the negativization of the expression of CD38 in PC, demonstrating a resistance mechanism under the clonal evolution theory. We report the case of a 63year-old male, diagnosed with a relapsed/refractory multiple myeloma, heavily treated, who expressed strong CD38 marker at the beginning of the treatment, with a posterior negativization of CD38 after four cycles of treatment with daratumumab.
\end{abstract}

Categories: Internal Medicine, Oncology, Hematology

Keywords: multiple myeloma, relapsed, refractory

\section{Introduction}

Multiple myeloma is a hematopoietic disease in which the clonal expansion of neoplastic plasma cells (PC) disrupts bone marrow microenvironment, related to a para-protein secreted (clonal gamma globulin), which ends up causing tissue damage. During the disease duration, different treatment types are used in an attempt to eradicate the clonal cells to ultimately cure the multiple myeloma. In the last decade, several monoclonal antibodies have been developed against specific targets within tumor cells, as the CD38 antibodies like daratumumab [1-3].

CD38 is a transmembrane glycoprotein type II which improves migration, adhesion cellular signaling over normal conditions in PC. Nevertheless, in multiple myeloma, an overexpression becomes a specific target to treat the disease. Daratumumab joins to the tail of the Fc portion of FC gamma receptors (FcyR), which are in the effector immune cells. It allows the induction of PC death through three mechanisms: complementdependent cytotoxicity, antibody-dependent cytotoxicity, and antibody-dependent cellular phagocytosis. Thus, the union of FcyR ultimately induces programmed death. The negativization of CD38 expression in PC is supposedly a resistant mechanism under clonal evolution. Also, different mechanisms of primary and acquired resistance related to $\mathrm{CD} 38$ expression have been documented [4,5].

Review began 03/04/2020 Review ended 04/05/2020 Published 04/10/2020

\section{(c) Copyright 2020}

The mechanism of daratumumab-induced resistance is not completely clear. However, multiple in vitro researches have demonstrated some potential causes of daratumumab resistance. It is important to have in mind how much CD38 expression exists given the pivotal trials showed a greater possibility to reach response with CD38 antibodies within patients with higher expression of CD38 in PC compared to those who have lesser, as a starting point for describing the origin of the resistance. On the other hand, the downregulation of CD38 expression may be the main cause of acquired resistance against previous highly treated patients.

In November 2015, FDA approved daratumumab for the treatment of relapsed multiple myeloma [6]. However, in 2019 its use was introduced as the front-line treatment in patients eligible to transplantation combined with lenalidomide (MAIA trial) [7]. In Colombia, INVIMA (Instituto Nacional de Vigilancia de Medicamentos y Alimentos) approved daratumumab for relapsed/refractory multiple myeloma after two lines of treatment which, previously, included a proteasome inhibitor and immunomodulatory imide drugs (ImiDs) (POLLUX and CASTOR trials) [8,9]. Nevertheless, it has been approved recently as the front-line treatment for non-transplantation eligible patients based on the results of a clinical trial (ALCYONE trial) [10]. This work has been published as an abstract (https://www.clinical-lymphoma-myelomaleukemia.com/article/S2152-2650(18)30885-1/abstract).

\section{Case Presentation}

A 63-year-old male patient diagnosed with IgA kappa multiple myeloma in 2011 treated with PAD regimen (Adriamycin, bortezomib, and dexamethasone) as front-line treatment. A complete response was reached 


\section{Cureus}

and posterior consolidation with autologous stem cell transplantation was performed. He suffered relapse after two years. Several rescue treatments were given without any response: lenalidomide + dexamethasone, CyBorD, carfilzomib + dexamethasone, cyclophosphamide + dexamethasone, VRD (bortezomib + lenalidomide + dexamethasone).

Just before the last relapse, flow cytometry showed 63.5\% of PC with an expression of CD38+ (CD38/CD138: 63.5\% and CD38/CD56: 63.5\%), CD138, CD56, beta-2 microglobulin, and cytoplasmic kappa light chain, CD19-, CD45- (Figure 1). Afterward, a daratumumab + lenalidomide + dexamethasone regimen was started.

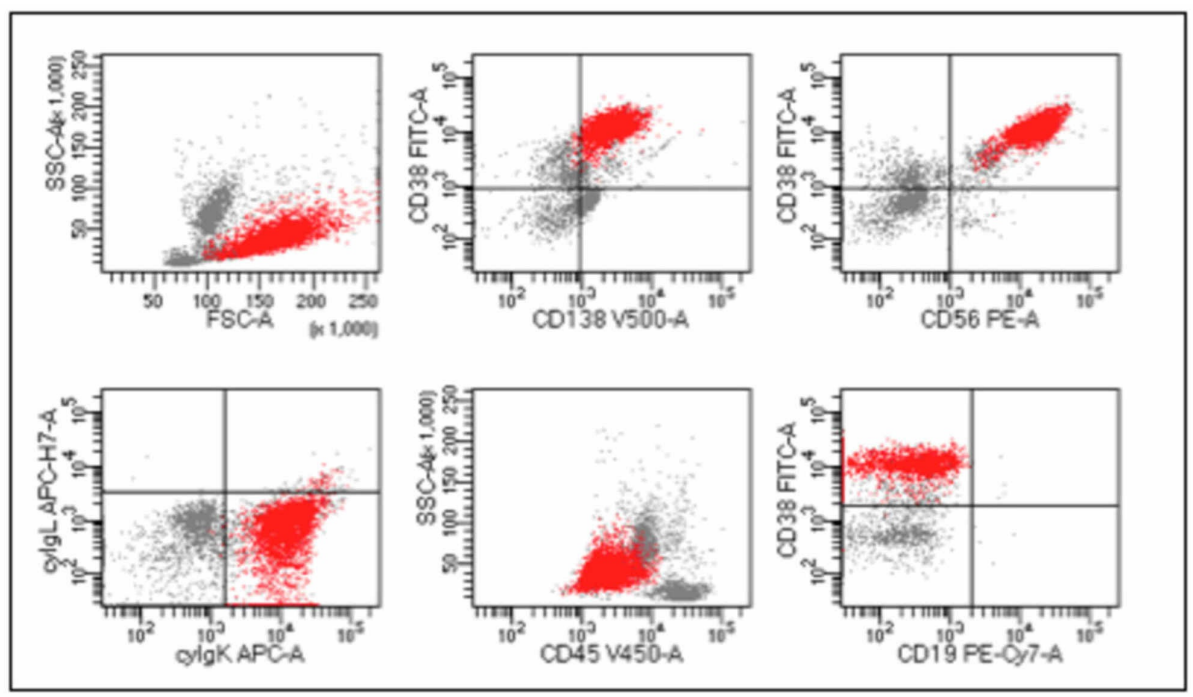

FIGURE 1: Flow cytometry showed $63.5 \%$ of myeloma cells (red events).

After four cycles of treatment, a response evaluation was performed documenting partial response. Nevertheless, flow cytometry documented $4.8 \%$ of abnormal PC in bone marrow with an expression of CD38- (CD38-/CD138+: 4.8\%, CD38-/CD56+: 4.8\%), CD138+, CD56+, beta-2 microglobulin, cytoplasmic kappa light chain, CD19-, CD45- (Figure 2).

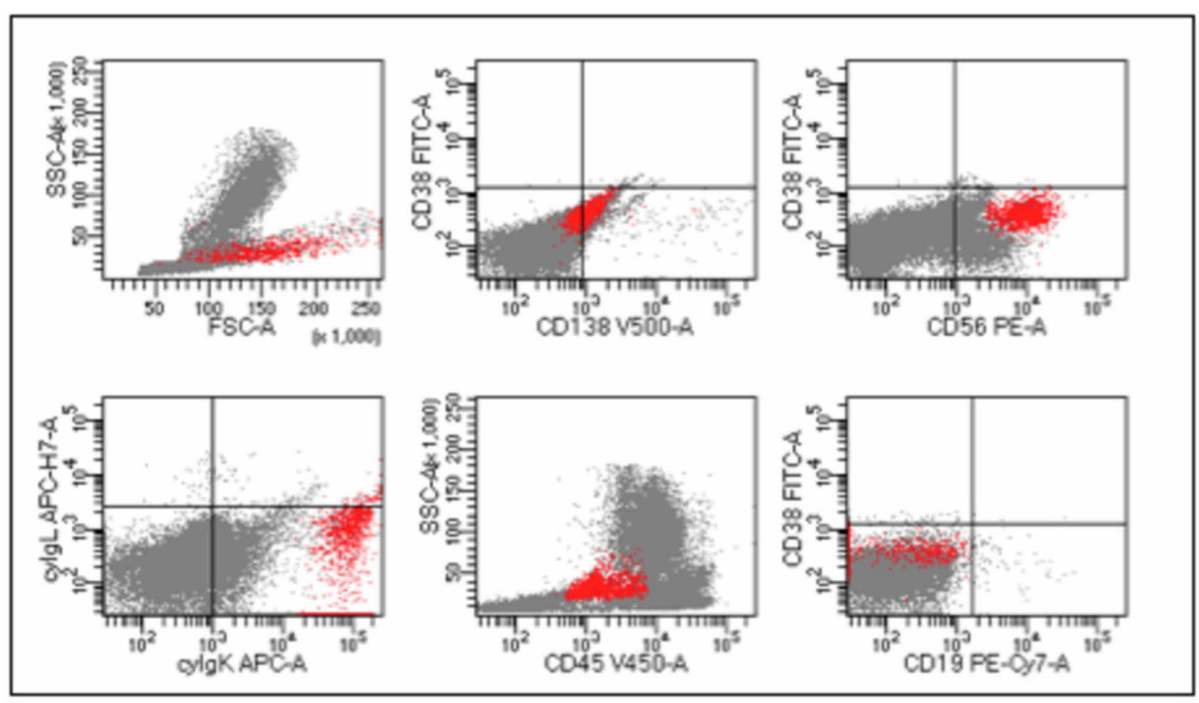

FIGURE 2: Flow cytometry showed $2.9 \%$ of myeloma cells (red events), CD38 negative.

Due to the sustained clinical and biochemical response, daratumumab was continued. However, the patient suffered severe pneumonia requiring management in the intensive care unit. Accordingly, daratumumab was interrupted. Pomalidomide + cyclophosphamide + dexamethasone was ordered but it was not authorized by the health insurance. 
Six months after daratumumab was ceased, the patient presented progression with M-protein rising and new bone lytic lesions. After multidisciplinary consideration, daratumumab was restarted with the same intensity of cycle 1 according to the POLLUX trial, plus antibiotic prophylaxis. CD38 was detected weak to negative expression by flow cytometry on bone marrow aspirate. Clinical improvement was documented early and the IgA levels reduced by $56 \%$ after just one cycle of re-challenge (Figure 3).

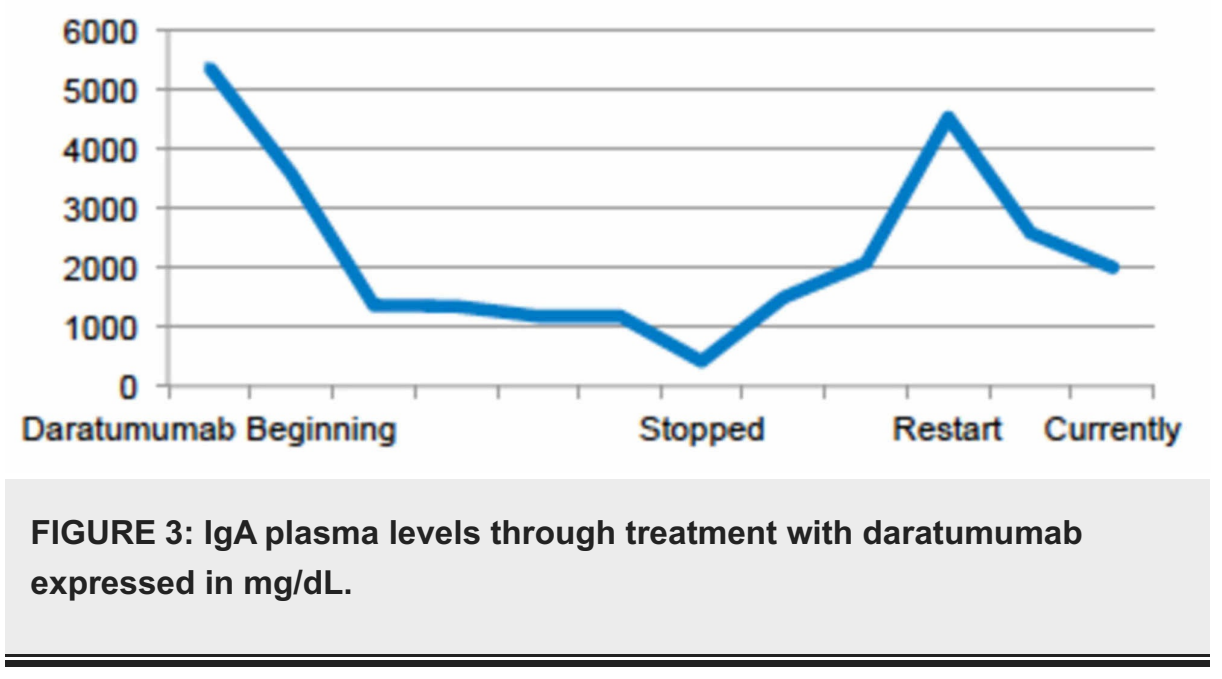

\section{Discussion}

Daratumumab activates cytotoxic immune effector, antibodies-dependent cytotoxicity, antibodies-mediated phagocytosis and complement-dependent cytotoxicity. Furthermore, it modulates CD38 enzyme reducing adenosine levels (which normally produces an immunosuppressor activity), enhancing the immune response against tumor $[1,3,5]$.

CD38 expression in PC is related to response to daratumumab treatment. Hence, we can divide the daratumumab into two categories: Primary and acquired resistance. Similar to other neoplasm treatments with monoclonal antibodies like Rituximab, Alemtuzumab, and Trastuzumab, the response depends on target antigen expression [11-14].

Treatment with daratumumab ends up also in a downregulation in myeloid-derived suppressor cells (MDSCs), regulatory T cell (Treg) and regulatory B cells (Bregs) activity. Furthermore, daratumumab produces and CD41 T-helper cells and CD81 cytotoxic T cells expansion. The measurement of IFN-Gamma levels indicates that due to T-cell clonality antiviral responses are increased after daratumumab treatment. These features indicate an appropriate T-cell response, despite low CD38 expression and may confirm T-cell response is due to depletion of regulatory cells. Those physiological changes are strong in patients who have shown any response to daratumumab, compared with those who did not. However, patients who have experienced relapse, showed loss of those features $[5,15]$.

Complement-dependent cytotoxicity is one of the most important mechanisms of action against PC with daratumumab. It has been demonstrated reduction of CD55 and CD59 expression enhances daratumumabmediated complement-dependent cytotoxicity in PC with high expression of $\mathrm{CD} 38$. In agreement, the expression of complement inhibitory proteins was not related to a response with daratumumab treatment, at least in monotherapy. Furthermore, CD55 and CD59 levels were higher in patients at the moment of progression compared with previous levels. A similar situation occurs in another neoplasm like chronic lymphocytic leukemia and rituximab, with this mechanism being a way of resistant for monoclonal antibodies due to impairment of complement-dependent cytotoxicity $[1,16]$.

CD38 expression was detected weak to negative with our patient. This downregulation by internalization of CD38 after daratumumab exposure may explain a way of resistance. Nevertheless, several mechanisms could be feasible in the response shown in this case. Firstly, daratumumab will eliminate PC with higher CD38 levels of expression. Downregulation of CD38 might be a way to acquiring resistance to evade the immune response triggered by daratumumab, generally by internalization. The downregulation is temporary because, after six months of daratumumab cessation, CD38 expression rose up in PC. It has been demonstrated in other research, as it occurs with other monoclonal antibodies like rituximab in hematological diseases $[1,2,4,17,18]$. In the case described above, after six months of cessation of treatment with daratumumab, partial response was achieved, with clear evidence of increasing expression of CD38 in PC and possible resensitization of PC to daratumumab.

Nevertheless, reduction of CD38 expression after daratumumab exposure is possible in both, patients with refractory diseases and those who respond to daratumumab. Maintenance of low expression of CD38 might 
be a way of resistance by PC; however, patients who have demonstrated a response with daratumumab despite lack of CD38 expression may have biologic plausibility due to physiologic ligands for CD38 and CD31 expressed in matrix component. PC with a higher CD38 expression tend to have more adherence to stromal cells of bone marrow probably via CD38-CD31 interaction. Decreasing PC adhesion to bone marrow accessory cells via blockage of CD31-38 interaction might be a new potential mechanism to reach antimultiple myeloma activity as well $[4,5,19]$.

Even though CD38 reduction is common after daratumumab treatment, and it is related to the possibility of response, the upregulation of CD38 molecules enhances the response option with daratumumab in resistant PC clones as we have demonstrated in this case report. Thus, other approach for patients to become PC sensitive to daratumumab is to treat them with all-trans retinoic acid (ATRA). ATRA increases CD38 expression of PC, restoring expression of this molecule, but also reduces CD55 and CD59 levels improving astonishingly the complement-dependent cytotoxicity as one of the most important mechanisms of action of daratumumab $[1,4]$.

\section{Conclusions}

The negativization of CD38 expression in PC might be an evidence of resistant mechanism under clonal evolution. However, different mechanisms of loss of expression have been described: primary (lack of expression) and acquired (downregulation expression or hide receptor due to monoclonal antibodies). Regarding the resistance, the mechanism is not completely clear, but several in vitro researches have demonstrated some potential causes of daratumumab resistance and re-sensitization. This is an interesting pathway of further investigation in multiple myeloma: the search for re-sensitization of PC to the treatments currently available, not just ATRA for daratumumab, but other drugs for multiple myeloma like IMiDs, and proteasome inhibitors, which are highly effective.

\section{Additional Information \\ Disclosures}

Human subjects: Consent was obtained by all participants in this study. Instituto Nacional de Cancerología issued approval NA. Consent was obtained by all the participants in this report. Instituto Nacional de Cancerología issued approval not applicable. Regarding the descriptive and retrospective cases where it has not been possible to obtain a patient's permission (in case of death or loss of contact), if a patient's confidentiality has been preserved and when the number of patients is less than ten, the ethics committee approves without an ethics meeting applying the manuscript as institutional statement. Conflicts of interest: In compliance with the ICMJE uniform disclosure form, all authors declare the following: Payment/services info: All authors have declared that no financial support was received from any organization for the submitted work. Financial relationships: All authors have declared that they have no financial relationships at present or within the previous three years with any organizations that might have an interest in the submitted work. Other relationships: All authors have declared that there are no other relationships or activities that could appear to have influenced the submitted work.

\section{References}

1. Nijhof IS, Groen RWJ, Lokhorst HM, et al.: Upregulation of CD38 expression on multiple myeloma cells by all-trans retinoic acid improves the efficacy of daratumumab. Leukemia. 2015, 29:2039-2049. 10.1038/leu.2015.123

2. van de Donk NWCJ, Usmani SZ: CD38 antibodies in multiple myeloma: mechanisms of action and modes of resistance. Front Immunol. 2018, 9:2134. 10.3389/fimmu.2018.02134

3. Overdijk MB, Verploegen S, Bögels M, et al.: Antibody-mediated phagocytosis contributes to the anti-tumor activity of the therapeutic antibody daratumumab in lymphoma and multiple myeloma. mAbs. 2015, 7:311320. 10.1080/19420862.2015.1007813

4. Nijhof IS, Casneuf T, van Velzen J, et al.: CD38 expression and complement inhibitors affect response and resistance to daratumumab therapy in myeloma. Blood. 2016, 128:959-970. 10.1182/blood-2016-03-703439

5. Krejcik J, Casneuf T, Nijhof IS, et al.: Daratumumab depletes CD38+ immune regulatory cells, promotes Tcell expansion, and skews T-cell repertoire in multiple myeloma. Blood. 2016, 128:384-394. 10.1182/blood2015-12-687749

6. Drug trials snapshots; DARZALEX. (2016). Accessed: March 03, 2020: https://www.fda.gov/drugs/drugapprovals-and-databases/drug-trials-snapshots-darzalex.

7. Facon T, Kumar S, Plesner T, et al.: Daratumumab plus lenalidomide and dexamethasone for untreated myeloma. N Engl J Med. 2019, 380:2104-2115. 10.1056/NEJMoa1817249

8. Dimopoulos MA, Oriol A, Nahi H, et al.: Daratumumab, lenalidomide, and dexamethasone for multiple myeloma. N Engl J Med. 2016, 375:1319-1331. 10.1056/NEJMoa1607751

9. Palumbo A, Chanan-Khan A, Weisel K, et al.: Daratumumab, bortezomib, and dexamethasone for multiple myeloma. N Engl J Med. 2016, 375:754-766. 10.1056/NEJMoa1606038

10. Mateos M-V, Dimopoulos MA, Cavo M, et al.: Daratumumab plus bortezomib, melphalan, and prednisone for untreated myeloma. N Engl J Med. 2018, 378:518-528. 10.1056/NEJMoa1714678

11. Lipton A, Köstler WJ, Leitzel K, et al.: Quantitative HER2 protein levels predict outcome in fluorescence in situ hybridization-positive patients with metastatic breast cancer treated with trastuzumab. Cancer. 2010, 116:5168-5178. 10.1002/cncr.25430

12. Lipton A, Goodman L, Leitzel K, et al.: HER3, p95HER2, and HER2 protein expression levels define multiple 


\section{Cureus}

subtypes of HER2-positive metastatic breast cancer. Breast Cancer Res Treat. 2013, 141:43-53.

10.1007/s10549-013-2665-0

13. Suzuki Y, Yoshida T, Wang G, et al.: Association of CD20 levels with clinicopathological parameters and its prognostic significance for patients with DLBCL. Ann Hematol. 2012, 91:997-1005. 10.1007/s00277-0121407-4

14. Rao SP, Sancho J, Campos-Rivera J, et al.: Human peripheral blood mononuclear cells exhibit heterogeneous CD52 expression levels and show differential sensitivity to alemtuzumab mediated cytolysis. PLoS One. 2012, 7:e39416. 10.1371/journal.pone.0039416

15. Lonial S, Weiss BM, Usmani SZ, et al.: Daratumumab monotherapy in patients with treatment-refractory multiple myeloma (SIRIUS): an open-label, randomised, phase 2 trial. Lancet. 2016, 387:1551-1560. 10.1016/S0140-6736(15)01120-4

16. Bannerji R, Kitada S, Flinn IW, Pearson M, Young D, ReedJohn JC, Byrd C: Apoptotic-regulatory and complement-protecting protein expression in chronic lymphocytic leukemia: relationship to in vivo rituximab resistance. J Clin Oncol. 2003, 21:1466-1471. 10.1200/JCO.2003.06.012

17. Berinstein NL, Grillo-López AJ, White C, et al.: Association of serum Rituximab (IDEC-C2B8) concentration and anti-tumor response in the treatment of recurrent low-grade or follicular non-Hodgkin's lymphoma. Ann Oncol. 1998, 9:995-1001. 10.1023/A:1008416911099

18. Oostendorp M, Lammerts van Bueren JJ, Doshi P, et al.: When blood transfusion medicine becomes complicated due to interference by monoclonal antibody therapy. Transfusion. 2015, 55:1555-1562. 10.1111/trf.13150

19. An G, Jiang H, Acharya C, et al.: SAR 650984, a therapeutic anti-CD38 monoclonal antibody, blocks CD38CD31 interaction in multiple myeloma. Blood. 2014, 124:4729. 10.1182/blood.V124.21.4729.4729 